\title{
Probing ultrafast coherent dynamics in core-excited xenon by using attosecond XUV-NIR transient absorption spectroscopy
}

\author{
Daria Kolbasova $\odot, 1,2,{ }^{*}$ Maximilian Hartmann $\odot,{ }^{3, \dagger}$ Rui Jin $\odot,{ }^{1}$ Alexander Blättermann, ${ }^{3}$ Christian Ott $\odot,{ }^{3}$ Sang-Kil Son $\odot,{ }^{1}$ \\ Thomas Pfeifer ${ }^{\circ},^{3}$ and Robin Santra ${ }^{1,2}$ \\ ${ }^{1}$ Center for Free-Electron Laser Science CFEL, Deutsches Elektronen-Synchrotron DESY, Notkestrasse 85, 22607 Hamburg, Germany \\ ${ }^{2}$ Department of Physics, Universität Hamburg, Notkestrasse 9-11, 22607 Hamburg, Germany \\ ${ }^{3}$ Max-Planck-Institut für Kernphysik, Saupfercheckweg 1, 69117 Heidelberg, Germany
}

(Received 22 December 2020; accepted 11 March 2021; published 6 April 2021)

\begin{abstract}
We investigate the capability of attosecond transient absorption spectroscopy to characterize the dynamics of inner-shell-excited systems. In the transient absorption spectroscopy setup considered, wave packets are prepared by an attosecond XUV pulse and probed by a femtosecond NIR pulse. By using this, we study coherent electron dynamics in core-excited xenon atoms. In particular, we clarify which aspects of the dynamics can be revealed when the wave packets are probed using an NIR pulse and analyze why the inner-shell hole dynamics is more difficult to probe than the dynamics of the excited electron. We perform a theoretical analysis of the transient absorption signal as a function of the time delay between the XUV pump and NIR probe pulses, treating the excitation pulse perturbatively and the probe pulse nonperturbatively. We also demonstrate that an additional NIR dressing field can dramatically influence the transient absorption spectrum. Our theoretical predictions are compared with experimental results, suggesting that a precise characterization of the NIR pulse is necessary for a qualitative and quantitative comparison.
\end{abstract}

DOI: 10.1103/PhysRevA.103.043102

\section{INTRODUCTION}

Transient absorption spectroscopy [1-4] is a powerful and versatile technique for studying real-time dynamics in the gas, liquid, solid, and plasma phases. In its simplest form, it is based on measuring, as a function of the pump-probe delay, the spectral properties of a probe pulse transmitted through a sample excited by a pump pulse [5-19]. Various physical processes can be studied, depending on the intensity and photon energy, and the specific combination of pump and probe pulses. Strong-field ionization by an optical pump pulse, when the extreme ultraviolet (XUV) pulse serves as a probe, provides spectroscopic information on the residual ion, such as ion quantum-state distributions [20,21] and orbital alignment $[22,23]$. At lower field strengths, when the XUV pulse is the pump and the optical field acts as the probe, laser-dressing and molecular alignment effects may be investigated [24-32].

The advent of attosecond light pulses [33-37] has opened up the possibility of studying fundamental questions related to quantum dynamics of electrons at their natural timescale. In particular, attosecond transient absorption spectroscopy (ATAS) [29,38-40] gives access to ultrafast dynamics of bound and autoionizing electronic states in atoms, molecules, and solids. An attosecond pulse coherently populates bright (dipole-allowed) electronic states, thus creating an electronic wave packet. Such a coherent superposition of quantum states gives rise to a dynamical evolution of the system being inves-

\footnotetext{
*Corresponding author: daria.kolbasova@cfel.de

†Corresponding author: maximilian.hartmann@mpi-hd.mpg.de
}

tigated. The characteristic timescales at which the system's physical properties vary correspond to the inverse of the frequency spacings among the coherently excited states of the wave packet. There have been a number of pump-probe studies investigating coherent valence- or Rydberg-electron dynamics in atoms [40-47]. The electronic coherence was observed under different conditions ranging from one-photon one-electron excitations, over multiphoton one-electron excitation, to one-photon two-electron excitation. In most scenarios, the key to probing the wave packet is lightinduced interference of the investigated states, which leads to characteristic modulation of the experimentally accessible quantity, e.g., the emitted photoelectrons or the transmitted photons. However, so far the characterization of electronic wave packets has been limited to the excited valence or Rydberg electron, whereas more deeply bound electrons (or the corresponding holes) can serve as important markers in siteselective and element-specific studies of complex targets.

Here we explore the possibility of the experimental observation of core-excited wave-packet coherence by ATAS of xenon. By using an attosecond XUV pulse we initiate a coreexcited wave packet. In order to probe the wave packet and monitor its evolution in time, we employ a time-delayed ultrashort laser pulse in the near-infrared (NIR) regime and detect the resulting modification of the transmitted XUV spectrum. The main goal of our study is to explore XUV-NIR transient absorption spectra and to elucidate whether excited-electron dynamics or inner-shell hole dynamics is encoded in the timeresolved spectra. In our previous work [48] we provided an elementary explanation of the signals one can obtain in ATAS experiments based on a perturbative treatment for both pump 
and probe pulses. In the current work, we present a nonperturbative treatment for the probe pulse to accommodate a wide range of experimentally accessible pulse properties. Finally, we compare our theoretical predictions with experiment.

\section{THEORY}

\section{A. Attosecond transient absorption spectroscopy}

Taking into account the rather small pulse energies of currently available attosecond sources, we perform our analysis of the transient absorption signal as a function of the time delay between the attosecond excitation pump pulse and the femtosecond probe pulse, treating the XUV pulse perturbatively. Unlike our previous work [48], we do not restrict ourselves to a weak probe field but treat it nonperturbatively, which allows us to capture nonlinear processes driven by the NIR field. Furthermore, numerical treatment of the NIR pulse enables us to employ the exact temporal shape of the pulse used in experiments, which is crucial for a quantitative comparison with the experimental data.

We employ the electric dipole approximation and assume that the pump and probe pulses are both linearly polarized along the $z$ axis. Following the logic presented in Ref. [38], we start by solving the time-dependent Schrödinger equation

$$
\begin{gathered}
i \frac{\partial}{\partial t}|\Psi, t\rangle=\hat{H}(t)|\Psi, t\rangle, \\
\hat{H}(t)=\hat{H}_{0}-E_{0}-\varepsilon_{d}(t) \hat{Z}-\varepsilon_{x}(t) \hat{Z},
\end{gathered}
$$

where $\hat{H}_{0}$ is the unperturbed Hamiltonian of the electronic system, $E_{0}$ is its ground-state energy, $\hat{Z}$ is the $z$ component of the electric dipole operator, and $\varepsilon_{d}(t)$ and $\varepsilon_{x}(t)$ are the amplitude of the dressing (NIR) and attosecond (XUV) electric fields, respectively. Assuming that the effect of the attosecond pulse can be treated perturbatively, the solution of Eq. (1) is

$$
|\Psi, t\rangle=\left|\Psi_{d}, t\right\rangle+\left|\Psi_{d}, t\right\rangle^{(1)}+\cdots,
$$

where $\left|\Psi_{d}, t\right\rangle$ is the optically dressed electronic state vector in the absence of the attosecond pulse and $\left|\Psi_{d}, t\right\rangle^{(1)}$ is the firstorder correction with respect to the attosecond pulse, evolving in the dressing field $\varepsilon_{d}(t)$. We employ the time-evolution operator $\hat{U}_{d}(t, \tau)$ to determine the optically dressed state vector $\left|\Psi_{d}, t\right\rangle$ :

$$
\left|\Psi_{d}, t\right\rangle=\hat{U}_{d}(t, \tau)\left|\Psi_{0}\right\rangle
$$

Here $\tau$ is a time before the system is optically dressed and $\left|\Psi_{0}\right\rangle$ is the initial state. The dipole moment of the electronic system along the $z$ axis can be expressed as

$$
D(t)=\langle\Psi, t|\hat{Z}| \Psi, t\rangle=D_{d}(t)+D^{(1)}(t)+\cdots .
$$

The term $D_{d}(t)$ describes harmonic generation driven by the dressing pulse only and $D^{(1)}(t)$ is the dipole moment correction to first order with respect to $\varepsilon_{x}(t)$ :

$$
\begin{gathered}
D_{d}(t)=\left\langle\Psi_{d}, t|\hat{Z}| \Psi_{d}, t\right\rangle \\
D^{(1)}(t)=i \int_{-\infty}^{t} d t^{\prime} \varepsilon_{x}\left(t^{\prime}\right)\left\langle\Psi_{d}, t\left|\hat{Z} \hat{U}_{d}\left(t, t^{\prime}\right) \hat{Z}\right| \Psi_{d}, t^{\prime}\right\rangle+\text { c.c. }
\end{gathered}
$$

\section{B. Wave-packet time-propagation method}

Assuming that the attosecond pulse is shorter than all relevant electronic timescales [38], we approximate it by a delta function $\varepsilon_{x}(t)=A_{x} \delta\left(t-t_{0}\right)$, where $t_{0}$ is the moment when the attosecond pulse interacts with the system. Hence,

$$
D^{(1)}(t)= \begin{cases}0, & t<t_{0} \\ i A_{x}\left\langle\Psi_{d}, t\left|\hat{Z} \hat{U}_{d}\left(t, t_{0}\right) \hat{Z}\right| \Psi_{d}, t_{0}\right\rangle+\text { c.c. }, & t \geqslant t_{0} .\end{cases}
$$

We will assume throughout that the dressing field $\varepsilon_{d}(t)$ is not strong enough to excite the ground state of the system. Therefore, $\left\langle\Psi_{d}, t\right| \approx\left\langle\Psi_{0}\right|$ and $\left|\Psi_{d}, t_{0}\right\rangle \approx\left|\Psi_{0}\right\rangle$. As a consequence,

$$
D^{(1)}(t)= \begin{cases}0, & t<t_{0} \\ i A_{x}\left\langle\tilde{\Psi}, t_{0}\left|\hat{U}_{d}\left(t, t_{0}\right)\right| \tilde{\Psi}, t_{0}\right\rangle+\text { c.c. }, \quad t \geqslant t_{0},\end{cases}
$$

where

$$
\left|\tilde{\Psi}, t_{0}\right\rangle=\hat{Z}\left|\Psi_{0}\right\rangle
$$

is the initial state of the electronic wave packet right after excitation by the XUV pump pulse at $t=t_{0}$. Thus, introducing the autocorrelation function

$$
C\left(t, t_{0}\right)=\left\langle\tilde{\Psi}, t_{0} \mid \tilde{\Psi}, t\right\rangle,
$$

where

$$
|\tilde{\Psi}, t\rangle=\hat{U}_{d}\left(t, t_{0}\right)\left|\tilde{\Psi}, t_{0}\right\rangle
$$

reflects the time evolution of the XUV-excited system in the dressing field, the dipole moment is given by

$$
D^{(1)}(t)= \begin{cases}0, & t<t_{0} \\ i A_{x} C\left(t, t_{0}\right)+\text { c.c., } & t \geqslant t_{0} .\end{cases}
$$

We compute the autocorrelation function $C\left(t, t_{0}\right)$ by employing the following numerical strategy. Unitary wavepacket propagation (in the absence of Auger decay) is ensured by approximating the relevant time-evolution operator for a time step from $t$ to $t+\Delta t$ through

$$
\hat{U}_{d}(t+\Delta t, t) \approx e^{-i \hat{H}_{d}(t+\Delta t / 2) \Delta t}
$$

which has an error that, to leading order, scales as $\Delta t^{3}$. Note that the Hamiltonian in Eq. (14),

$$
\hat{H}_{d}(t)=\hat{H}_{0}-E_{0}-\varepsilon_{d}(t) \hat{Z},
$$

does not include the XUV field.

As described in more detail in Sec. II C, we calculate a finite set of inner-shell-excited eigenstates $\left\{\left|\Psi_{I}\right\rangle\right\}$ and expand the inner-shell-excited wave packet in this subspace:

$$
|\tilde{\Psi}, t\rangle=\sum_{I} a_{I}(t)\left|\Psi_{I}\right\rangle
$$

Upon combining Eqs. (14) and (16), we obtain an expression for the propagation of the vector of expansion coefficients

$$
\boldsymbol{a}(t+\Delta t)=e^{-i \boldsymbol{H}_{d}(t+\Delta t / 2) \Delta t} \boldsymbol{a}(t),
$$

where $(\boldsymbol{a})_{I}=a_{I}$ and $\left(\boldsymbol{H}_{d}\right)_{I J}=\left\langle\Psi_{I}\left|\hat{H}_{d}\right| \Psi_{J}\right\rangle$, with $I$ and $J$ indices that run from 1 to $N$, where $N$ is the size of the finite set.

We solve Eq. (17) using the initial condition

$$
a_{I}\left(t_{0}\right)=\left\langle\Psi_{I} \mid \tilde{\Psi}, t_{0}\right\rangle=\left\langle\Psi_{I}|\hat{Z}| \Psi_{0}\right\rangle .
$$


Diagonalization of the instantaneous Hamiltonian matrix in each time step is done in MATLAB [49], which uses LAPACK routines [50] to compute the eigenvalues $\tilde{E}_{k}$ and eigenvectors $\boldsymbol{v}_{k}$. Hence,

$$
\boldsymbol{a}(t+\Delta t)=\sum_{k} e^{-i \tilde{E}_{k}(t+\Delta t / 2) \Delta t} \boldsymbol{v}_{k}(t+\Delta t) \boldsymbol{v}_{k}^{\dagger}(t+\Delta t) \boldsymbol{a}(t) .
$$

Via

$$
C\left(t, t_{0}\right)=\sum_{I} a_{I}^{*}\left(t_{0}\right) a_{I}(t)
$$

we obtain the autocorrelation function required for the evaluation of Eq. (13).

Once the XUV-induced dipole moment in the presence of laser dressing is available, the XUV cross section can be calculated

$$
\sigma(\omega)=\frac{4 \pi \omega}{c} \operatorname{Im}\left[\frac{\tilde{D}^{(1)}(\omega)}{\tilde{\varepsilon}_{x}(\omega)}\right],
$$

where $\tilde{D}^{(1)}(\omega)$ and $\tilde{\varepsilon}_{x}(\omega)$ are the Fourier transforms of $D^{(1)}(t)$ and $\varepsilon_{x}(t)$, respectively. The computed XUV cross sections presented in Sec. III are converged with respect to the time step $\Delta t$ and with respect to the basis set $\left\{\left|\Psi_{I}\right\rangle\right\}$.

\section{Calculation of atomic parameters for $\mathrm{Xe}$}

To build a reliable atomic model it is crucial to use realistic parameters for the states involved. Even though there are many experimental data available regarding electromagnetic transitions in various atomic species, these data are not sufficiently complete. Therefore, we employ first-principles atomic-structure software to calculate the missing information.

In the present work, we employ flexible atomic code (FAC) [51] to calculate the atomic structure and transition dipole matrix elements. We construct a set of orbitals based on the Dirac-Fock-Slater effective local potential for the ground configuration of neutral $\mathrm{Xe}$ and diagonalize the Hamiltonian matrices for core-excited states that are constructed from initial and final nonrelativistic electron configurations.

We build our model by using energies from experiment [52,53], whenever available, and complementing them with energies from FAC calculations. Only a few Xe $4 d$ excited dark states have been observed experimentally. For those known levels, our FAC calculations differ almost uniformly from experimental values by $\sim 1.7 \mathrm{eV}$, so we shift all calculated energies accordingly. All transition dipole moments are taken from our FAC calculations.

In our Xe model we distinguish between bright (dipoleallowed) and dark (dipole-forbidden) states with respect to excitation from the ground state. We include the states with principal quantum number $n \leqslant 8$ for the bright and $n \leqslant 20$ for the dark states and confirm that our results are converged with them. The parameters of the states are listed in Table I (a subset of the dark states are listed for brevity). Assuming the lifetime of all excited states to be defined by the Auger decay of a $4 d$ hole, we replace the energy eigenvalues $\tilde{E}_{k}$ in Eq. (19) with $\tilde{E}_{k}-i \Gamma / 2$. Here $\Gamma=0.111 \mathrm{eV}$ is the decay width of a $4 d$ hole, corresponding to a lifetime of $\sim 6$ fs [54]. Thus, the state vector $|\tilde{\Psi}, t\rangle$ that underlies the autocorrelation function $C\left(t, t_{0}\right)$ undergoes exponential decay.

As a consequence of its large spectral bandwidth, the attosecond XUV pump pulse can excite the Xe atom from its ground state $J^{P}=0^{+}$to any of the bright states $J^{P}=1^{-}$, creating a wave packet with a high degree of coherence. We assume the probe laser field not to be strong enough to affect the Xe ground state. However, it can couple a bright state $1^{-}$ to a dark state $J^{+}$, by exchanging one NIR photon with the atom, or it can couple to another bright state $1^{-}$by exchanging two NIR photons. Since the ground state $0^{+}$and the dark states $J^{+}$have the same parity, the latter cannot be excited via one-photon absorption from the ground state (i.e., the corresponding transition dipole moments are zero); however, in the presence of the dressing laser field, they can give rise to accessible light-induced states (LISs) [55-57]. The LISs in the absorption spectrum are then interpreted, in the frequency domain, as the intermediate states of processes that involve the absorption or emission of an additional dressing NIR photon.

In Fig. 1 we show the computed XUV cross section of Xe without any additional electromagnetic field (gray curve) and dressed by a field with a photon energy of $\sim 0.7 \mathrm{eV}$ and a peak intensity of $\sim 5 \times 10^{10} \mathrm{~W} / \mathrm{cm}^{2}$ (black curve). In the absence of a dressing field, the attosecond XUV pulse coherently populates the Xe bright states $4 d^{9} n p^{1}(n \geqslant 6)$. When the atom gets dressed by the laser field, transitions to LISs become available. Dipole-allowed LISs can be observed as additional absorption lines. When the bright and dark states are not one-IR-photon resonant with each other, the LIS energy is one IR photon away from the dark state. However, when they are in resonance, the result is an Autler-Townes doublet, where the energy is determined light-induced Rabi splitting [58]. Similarly, in the nonresonant case, there are in general AC Stark shifts. In Fig. 1(b) the arrows show the parent dark state for the most important LISs. Accessibility of LISs and their corresponding particle-hole wave-packet dynamics depends on the phase properties of the NIR laser field, which provides rich manifolds of dynamics to be explored with time-delayed ATAS.

\section{RESULTS}

\section{A. Wave-packet dynamics induced by XUV only}

From the dependence of the XUV transient-absorption cross section $\sigma(\omega, \tau)$ on the time delay $\tau$ between the attosecond XUV and the NIR laser field, one can study the dynamics of inner-shell-excited particle-hole wave packets induced by the XUV pulse (superpositions of odd-parity states only). In the following, we take the Fourier transform of $\sigma(\omega, \tau)$ with respect to $\tau$. Figure 2 shows $2 \mathrm{D}$ maps of the square root of the resulting XUV one-photon cross section $\sqrt{\left|\tilde{\sigma}\left(\omega, \omega^{\prime}\right)\right|}$ for photon energies $\omega$ that cover the main $4 d$ excited states. Here and below $\omega^{\prime}$ is the Fourier energy associated with $\tau$.

As a probe field, we use a Gaussian-shaped NIR field

$$
\varepsilon_{d}(t)=A_{I R} \operatorname{Re}\left[e^{-\left[4 \ln (2) / \chi_{I R}^{2}\right]\left(t-t_{0}\right)^{2}} e^{i \omega_{I R} t}\right],
$$

with an amplitude of $A_{I R}=0.0012$ a.u. The central frequency $\omega_{I R}$ is chosen as $0.7 \mathrm{eV}$ in Figs. 2(a)-2(c) and $1.7 \mathrm{eV}$ in Figs. 2(d)-2(f) and the pulse duration $\chi_{I R}$ is one optical cycle 
TABLE I. Energies and transition dipole moments of the $4 d$ excited states of Xe used in our ATAS calculations, significant for convergence. The states listed below the ground state (GS) are dark states, whereas the states listed horizontally in the first row are $J^{P}=1^{-}$bright states. The values in parentheses are for $n p_{1 / 2}$; otherwise they are for $n p_{3 / 2}$.

\begin{tabular}{|c|c|c|c|c|c|c|c|c|c|c|}
\hline \multirow{2}{*}{$\begin{array}{l}\text { State } J^{P} \\
\text { GS }\end{array}$} & \multirow{2}{*}{$\frac{E(\mathrm{eV})}{0}$} & \multirow{2}{*}{$\begin{array}{c}4 d_{5 / 2}^{-1} 6 p^{1} \\
65.110 \\
-0.182\end{array}$} & \multicolumn{2}{|c|}{$\begin{array}{c}4 d_{3 / 2}^{-1} 6 p^{1} \\
67.039\end{array}$} & \multirow{2}{*}{$\begin{array}{c}4 d_{5 / 2}^{-1} 7 p^{1} \\
66.375 \\
-0.106\end{array}$} & \multicolumn{2}{|c|}{$\begin{array}{c}4 d_{3 / 2}^{-1} 7 p^{1} \\
68.345\end{array}$} & \multirow{2}{*}{$\begin{array}{r}4 d_{5 / 2}^{-1} 8 p^{1} \\
67.150 \\
-0.072\end{array}$} & \multicolumn{2}{|c|}{$\begin{array}{c}4 d_{3 / 2}^{-1} 8 p^{1} \\
69.150\end{array}$} \\
\hline & & & -0.138 & $(0.052)$ & & -0.015 & $(-0.083)$ & & -0.020 & $(-0.054)$ \\
\hline $4 d_{3 / 2}^{-1} 6 s_{1 / 2}^{1} 2^{+}$ & 63.707 & -0.039 & -8.989 & $(3.521)$ & 0.0 & -0.032 & $(-0.796)$ & 0.0 & -0.042 & $(-0.302)$ \\
\hline $4 d_{5 / 2}^{-1} 6 s_{1 / 2}^{1} 2^{+}$ & 65.670 & 9.548 & 0.075 & $(0.092)$ & 1.047 & 0.0 & $(0.0)$ & 0.444 & 0.0 & $(0.0)$ \\
\hline $4 d_{3 / 2}^{-1} 7 s_{1 / 2}^{1} 2^{+}$ & 65.830 & 0.088 & -8.029 & $(2.757)$ & -0.038 & -3.805 & $(-19.266)$ & 0.001 & -0.466 & $(-1.829)$ \\
\hline $4 d_{5 / 2}^{-1} 7 s_{1 / 2}^{1} 2^{+}$ & 67.878 & 9.200 & -0.127 & $(-0.066)$ & 19.236 & -0.069 & $(0.050)$ & 2.324 & -0.003 & $(0.0)$ \\
\hline $4 d_{3 / 2}^{-1} 8 s_{1 / 2}^{1} 2^{+}$ & 66.539 & 0.022 & -1.810 & $(0.695)$ & 0.061 & -2.735 & $(-17.592)$ & -0.034 & -11.786 & $(-30.662)$ \\
\hline $4 d_{5 / 2}^{-1} 8 s_{1 / 2}^{1} 2^{+}$ & 68.587 & 1.948 & -0.029 & $(-0.013)$ & 19.052 & 0.062 & $(-0.078)$ & 32.021 & -0.045 & $(0.050)$ \\
\hline $4 d_{3 / 2}^{-1} 9 s_{1 / 2}^{1} 2^{+}$ & 66.858 & 0.012 & -0.944 & $(0.369)$ & 0.014 & -0.703 & $(-3.565)$ & 0.046 & -9.644 & $(-28.448)$ \\
\hline $4 d_{5 / 2}^{-1} 9 s_{1 / 2}^{1} 2^{+}$ & 68.907 & 1.004 & -0.016 & $(-0.007)$ & 3.561 & 0.011 & $(-0.016)$ & 31.964 & 0.037 & $(-0.067)$ \\
\hline $4 d_{3 / 2}^{-1} 10 s_{1 / 2}^{1} 2^{+}$ & 67.029 & 0.008 & -0.620 & $(0.244)$ & 0.007 & -0.367 & $(-1.793)$ & 0.010 & -2.084 & $(-5.374)$ \\
\hline $4 d_{5 / 2}^{-1} 10 s_{1 / 2}^{1} 2^{+}$ & 69.078 & 0.657 & -0.010 & $(-0.004)$ & 1.765 & 0.006 & $(-0.008)$ & 5.581 & 0.006 & $(-0.013)$ \\
\hline $4 d_{5 / 2}^{-1} 5 d_{3 / 2}^{1} 2^{+}$ & 65.439 & 1.051 & 0.0 & $(0.0)$ & 1.622 & 0.005 & $(-0.008)$ & 2.274 & 0.002 & $(-0.006)$ \\
\hline $4 d_{5 / 2}^{-1} 5 d_{5 / 2}^{1} 2^{+}$ & 65.439 & 4.693 & 0.0 & $(0.0)$ & 7.310 & 0.023 & $(-0.037)$ & 10.330 & 0.010 & $(-0.027)$ \\
\hline $4 d_{3 / 2}^{-1} 5 d_{5 / 2}^{1} 2^{+}$ & 65.647 & 0.098 & -4.935 & $(-2.958)$ & 0.052 & 5.944 & $(-6.669)$ & 0.034 & 6.723 & $(-10.642)$ \\
\hline $4 d_{3 / 2}^{-1} 5 d_{3 / 2}^{1} 2^{+}$ & 67.408 & -0.029 & -0.513 & (5.785) & 0.0 & -8.621 & $(-2.275)$ & 0.0 & -12.389 & $(-0.935)$ \\
\hline $4 d_{5 / 2}^{-1} 5 d_{5 / 2}^{1} 0^{+}$ & 67.410 & 3.624 & 0.0 & $(0.0)$ & 5.637 & 0.018 & $(-0.028)$ & 7.956 & 0.008 & $(-0.019)$ \\
\hline $4 d_{3 / 2}^{-1} 5 d_{3 / 2}^{1} 0^{+}$ & 67.513 & -0.043 & 3.149 & $(-1.040)$ & -0.022 & 0.629 & $(5.027)$ & -0.014 & 1.960 & (6.746) \\
\hline $4 d_{5 / 2}^{-1} 6 d_{3 / 2}^{1} 2^{+}$ & 66.495 & 0.252 & 0.0 & $(-0.001)$ & -2.938 & 0.0 & $(-0.029)$ & -1.495 & 0.0 & $(0.019)$ \\
\hline $4 d_{5 / 2}^{-1} 6 d_{5 / 2}^{1} 2^{+}$ & 66.495 & 2.068 & -0.030 & $(-0.010)$ & -21.661 & -0.023 & $(0.082)$ & -10.576 & 0.0 & $(0.0)$ \\
\hline $4 d_{3 / 2}^{-1} 6 d_{5 / 2}^{1} 2^{+}$ & 66.495 & 0.038 & -2.063 & $(-1.384)$ & -0.115 & -17.572 & (20.009) & 0.016 & -6.607 & (11.368) \\
\hline $4 d_{3 / 2}^{-1} 6 d_{3 / 2}^{1} 2^{+}$ & 68.475 & -0.013 & -0.045 & $(2.271)$ & 0.025 & 26.151 & $(9.130)$ & -0.001 & 14.159 & (3.112) \\
\hline $4 d_{5 / 2}^{-1} 6 d_{5 / 2}^{1} 0^{+}$ & 68.475 & 1.565 & -0.007 & $(-0.014)$ & -16.526 & -0.093 & $(-0.147)$ & -8.097 & 0.056 & $(0.095)$ \\
\hline $4 d_{3 / 2}^{-1} 6 d_{3 / 2}^{1} 0^{+}$ & 68.475 & 0.005 & 1.230 & $(-0.302)$ & -0.167 & -2.861 & $(-15.956)$ & 0.100 & -3.272 & $(-7.981)$ \\
\hline $4 d_{5 / 2}^{-1} 7 d_{3 / 2}^{1} 2^{+}$ & 66.750 & 0.371 & -0.004 & $(-0.002)$ & 0.688 & 0.001 & $(-0.001)$ & -9.928 & -0.004 & $(-0.017)$ \\
\hline $4 d_{5 / 2}^{-1} 7 d_{5 / 2}^{1} 2^{+}$ & 66.755 & 1.355 & -0.021 & $(-0.008)$ & 2.617 & 0.005 & $(-0.012)$ & -34.973 & -0.002 & $(0.064)$ \\
\hline $4 d_{3 / 2}^{-1} 7 d_{5 / 2}^{1} 2^{+}$ & 66.769 & 0.028 & -1.402 & $(-1.053)$ & 0.016 & 2.515 & $(-2.204)$ & -0.085 & -27.138 & (36.900) \\
\hline $4 d_{3 / 2}^{-1} 7 d_{3 / 2}^{1} 2^{+}$ & 68.801 & 0.0 & -0.240 & $(1.523)$ & -0.004 & -2.564 & $(-0.616)$ & 0.010 & 43.188 & (10.952) \\
\hline $4 d_{5 / 2}^{-1} 7 d_{5 / 2}^{1} 0^{+}$ & 68.807 & 1.057 & -0.010 & $(-0.009)$ & 2.025 & 0.006 & $(0.002)$ & -27.479 & -0.104 & $(-0.139)$ \\
\hline $4 d_{3 / 2}^{-1} 7 d_{3 / 2}^{1} 0^{+}$ & 68.813 & -0.004 & 0.881 & $(-0.260)$ & 0.009 & 0.0 & (1.536) & -0.172 & -9.172 & $(-25.357)$ \\
\hline $4 d_{5 / 2}^{-1} 8 d_{3 / 2}^{1} 2^{+}$ & 66.977 & 0.940 & -0.015 & $(-0.006)$ & 1.758 & 0.004 & $(-0.008)$ & 3.157 & 0.002 & $(-0.007)$ \\
\hline $4 d_{5 / 2}^{-1} 8 d_{5 / 2}^{1} 2^{+}$ & 66.975 & 0.325 & -0.005 & $(-0.002)$ & 0.597 & 0.001 & $(-0.002)$ & 1.030 & 0.0 & $(-0.001)$ \\
\hline $4 d_{3 / 2}^{-1} 8 d_{5 / 2}^{1} 2^{+}$ & 66.986 & 0.020 & -0.990 & $(-0.780)$ & 0.012 & 1.773 & $(-1.530)$ & 0.009 & 2.709 & $(-3.140)$ \\
\hline $4 d_{3 / 2}^{-1} 8 d_{3 / 2}^{1} 2^{+}$ & 69.025 & 0.0 & -0.223 & (1.071) & 0.0 & -1.816 & $(-0.656)$ & -0.002 & -2.976 & $(-0.137)$ \\
\hline $4 d_{5 / 2}^{-1} 8 d_{5 / 2}^{1} 0^{+}$ & 69.029 & 0.749 & -0.009 & $(-0.006)$ & 1.394 & 0.005 & $(-0.001)$ & 2.477 & 0.004 & $(0.002)$ \\
\hline $4 d_{3 / 2}^{-1} 8 d_{3 / 2}^{1} 0^{+}$ & 69.033 & -0.005 & 0.635 & $(-0.197)$ & 0.0 & 0.091 & (1.163) & 0.008 & 0.211 & $(1.751)$ \\
\hline
\end{tabular}

$T=2 \pi / \omega_{I R}$ in Figs. 2(a) and 2(d), two times $T$ in Figs. 2(b) and 2(e), and six times $T$ in Figs. 2(c) and 2(f), where $T=$ $2.4 \mathrm{fs}$ for $\omega_{I R}=1.7 \mathrm{eV}$ and $T=6.0 \mathrm{fs}$ for $\omega_{I R}=0.7 \mathrm{eV}$.

The dynamics of inner-shell-excited particle-hole wave packets induced by the XUV pulse leads to oscillations of the atomic cross section as a function of the time delay with Fourier energies $\omega^{\prime}$ corresponding to the energy differences among the interfering states of the wave packet. This interference appears as diagonal features from one odd-parity state to another on the 2D map $\sqrt{\left|\tilde{\sigma}\left(\omega, \omega^{\prime}\right)\right|}$ in Figs. 2(a) and 2 (d). The peaks around $\omega^{\prime} \approx 1.3 \mathrm{eV}$ correspond to the interference of bright states with the same angular momentum $j$ of the $4 d$ hole but different principal quantum number of the Rydberg $p$ electron ( $n=6$ and 7), i.e., the difference between $4 d_{5 / 2}^{-1} 6 p^{1}$ and $4 d_{5 / 2}^{-1} 7 p^{1}(66.375-65.110=1.265 \mathrm{eV})$ and between $4 d_{3 / 2}^{-1} 6 p^{1}$ and $4 d_{3 / 2}^{-1} 7 p^{1}(68.345-67.039=1.306 \mathrm{eV})$. On the other hand, the peaks around $\omega^{\prime} \approx 2 \mathrm{eV}$ result from the interference of bright states with different angular momentum $j$ of the $4 d$ hole but the same $n$ of the excited $p$ electron (for example, $1.929 \mathrm{eV}$ for $n=6$ and $1.970 \mathrm{eV}$ for $n=7$ ). The latter peaks are strongly suppressed relative to the former peaks 


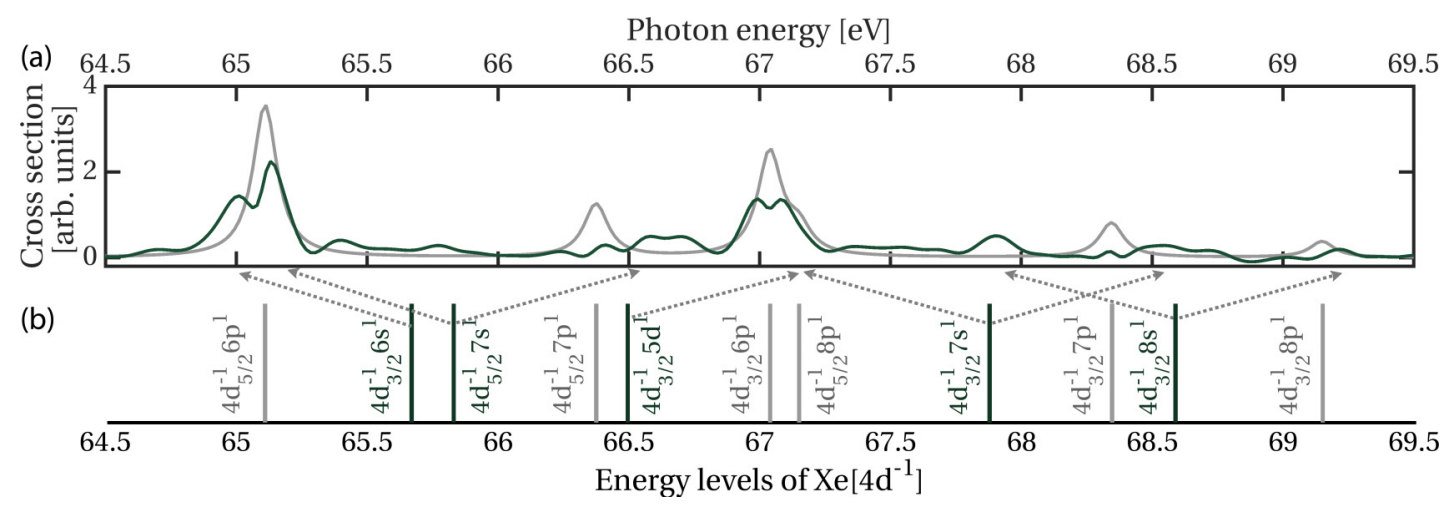

FIG. 1. (a) XUV cross section of Xe in the absence (gray) and presence (black) of a dressing field $\omega_{I R}=0.7 \mathrm{eV}$ and (b) energy levels of Xe with one excited $4 d$ electron (gray denotes bright states and black dark states). Arrows designate which dark states correspond to the most important LISs.

due to weak coupling between states with different angular momentum $j$ of the $4 d$ hole. Due to dipole selection rules, the transition from one odd-parity state to another requires an even number of photons. Therefore, the resonances $\omega^{\prime} \approx 1.3$ and $2 \mathrm{eV}$ are more pronounced in Fig. 2(a), where we use the photon energy $\omega_{I R}=0.7 \mathrm{eV}$, than in Fig. $2(\mathrm{~d})\left(\omega_{I R}=1.7 \mathrm{eV}\right)$, as $2 \omega_{I R}$ is closer to those resonances.

The strong peaks around $\omega^{\prime} \approx 3 \mathrm{eV}$ in Figs. 2(d)-2(f) rather appear as a horizontal feature. Although one can see in Fig. 2(d) the diagonals between the states $4 d_{5 / 2}^{-1} 6 p^{1}(\omega=$ $65.110 \mathrm{eV})$ and $4 d_{3 / 2}^{-1} 7 p^{1}(\omega=68.345 \mathrm{eV})$, for the state $4 d_{3 / 2}^{-1} 6 p^{1}(\omega=67.039 \mathrm{eV})$ there is no such diagonal, because there is no coupled bright state at $2 \omega_{N I R}$ away (i.e., neither at $64 \mathrm{eV}$ nor at $70 \mathrm{eV}$ is there a coupling partner included in the simulation). However, its cross section also reveals fairly strong $\omega^{\prime} \approx 3 \mathrm{eV}$ oscillations with the time delay. The reason is the following. When the duration of the probe pulse becomes longer than the lifetime of the $4 d$ excited state, the observation of the inner-shell-excited particle-hole wave-packet dynamics induced by the XUV becomes more difficult. The laser field creates active valence-electron dynamics throughout its duration, which strongly affects the atomic cross section for all absorption peaks. Accessibility of those states directly depends on the laser field potential, which leads to the horizontal features on the 2D map of $\sqrt{\left|\tilde{\sigma}\left(\omega, \omega^{\prime}\right)\right|}$ in Figs. 2(c) and 2(f). The dominant frequency of the cross-section oscillations as a function of the time delay between the XUV and NIR pulses becomes $\omega^{\prime}=2 n \omega_{I R}$ $(n=1,2, \ldots)$. Thus, the horizontal features are around $\omega^{\prime} \approx$ $1.4 \mathrm{eV}$ in Fig. 2(c) and $\omega^{\prime} \approx 3.4 \mathrm{eV}$ in Fig. 2(f). This tendency already appears for the pulse duration of $2 T$ in Figs. 2(b) and 2(e). These horizontal $2 \omega$ structures become broader with shorter pulses due to the larger Fourier bandwidth.

In Fig. 3 the Fourier spectrum of the main absorption peak $4 d_{5 / 2}^{-1} 6 p^{1}(\omega \approx 65.1 \mathrm{eV})$ is presented for a probe-pulse duration of $4 T$. Figure 3(a) corresponds to $\omega_{I R}=0.9 \mathrm{eV}$ and Fig. 3(b) to $\omega_{I R}=0.7 \mathrm{eV}$. These energies $\omega_{I R}$ of the laser field were chosen in order to create more interesting
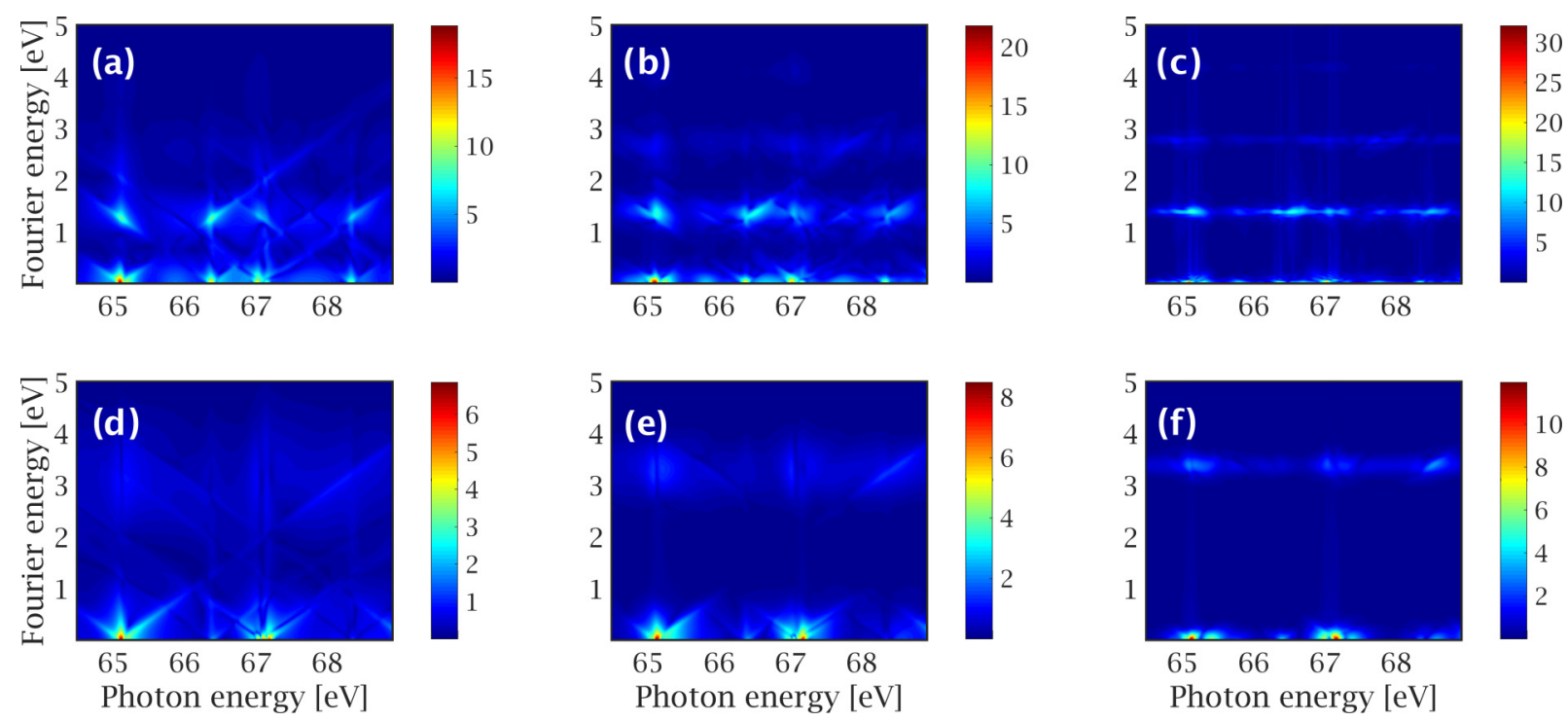

FIG. 2. Square root of the Fourier-transform amplitude of the XUV cross section $\sigma(\omega, \tau)$ of Xe $\left[\sqrt{\left|\tilde{\sigma}\left(\omega, \omega^{\prime}\right)\right|}\right]$. The photon energy of the probe field is (a)-(c) $\omega_{I R}=0.7 \mathrm{eV}$ and (d)-(f) $\omega_{I R}=1.7 \mathrm{eV}$. The duration of the probe field is (a) and (d) one period of the field $T$, (b) and (e) two periods $2 T$, and (c) and (f) $6 T$. 

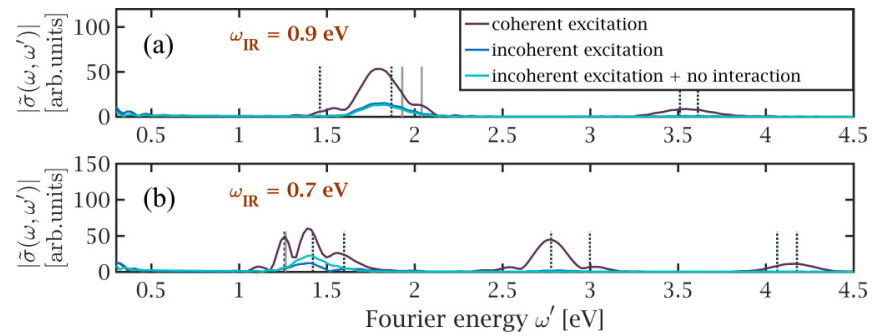

FIG. 3. Fourier-transformed XUV cross section $\left|\tilde{\sigma}\left(\omega, \omega^{\prime}\right)\right|$ for the $4 d_{5 / 2}^{-1} 6 p^{1}$ state of $\mathrm{Xe}$ at $\omega=65.1 \mathrm{eV}$. The duration of the laser pulse is $4 T$ and the photon energy $\omega_{I R}$ is (a) $0.9 \mathrm{eV}$ and (b) $0.7 \mathrm{eV}$. Gray vertical lines are used to mark bright-state-bright-state resonances and black dashed lines for LIS-bright-state resonances.

wave-packet dynamics and to be able to observe more resonances $\omega^{\prime} \approx 2 n \omega_{I R}(n=1,2, \ldots)$. Different colors are used for three different cases: (i) coherent excitation; (ii) "incoherent" excitation, where only the $4 d_{5 / 2}^{-1} 6 p^{1}$ state is allowed to be excited by the XUV field; and (iii) only $4 d_{5 / 2}^{-1} 6 p^{1}$ is allowed to be excited by the XUV and no interactions are allowed between the bright states through the dark states. Relatively short probe-pulse durations provide an energy spectrum that is sufficiently broad to observe inner-shell-excited wave-packet dynamics induced by the XUV. The gray vertical lines mark the bright-bright resonance positions for the $4 d_{5 / 2}^{-1} 6 p^{1}$ state. The black dashed lines represent the bright-state-LIS transitions. Although the bright-LIS interference peak at $\omega^{\prime}=$ $2 \omega_{I R}$ is dominant for both values of $\omega_{I R}$, the bright-bright interference peaks at $\omega^{\prime}=2 \mathrm{eV}$ [Fig. 3(a)] and $\omega^{\prime}=1.3 \mathrm{eV}$ [Fig. 3(b)] can still be seen under coherent excitation. For incoherent excitation, those bright-bright interference peaks are absent.

The main challenge with detecting the inner-shell hole dynamics is that its impact on the NIR-modulated XUV cross section is much weaker than the impact of the Rydbergelectron dynamics induced by the probe field. By reducing the duration of the NIR laser field one can decrease the effect of the latter dynamics, but it will also increase the width of the interference peaks in the Fourier spectrum. In order to observe inner-shell hole dynamics, the optimal laser pulse duration is comparable to the lifetime of the excited state $\left(\chi_{I R} \approx 1 / \Gamma\right)$. Also, the NIR photon energy needs to be close to the resonance $\omega_{I R}=\left|E_{1}^{-}-E_{2}^{-}\right| / 2$, where $E_{1}^{-}$and $E_{2}^{-}$are the energies of two bright states with different inner-shell holes (but the same state for the Rydberg electron). Another important requirement for observing inner-shell hole dynamics is sufficiently large dipole moments between the $E_{1}^{-}$and $E_{2}^{-}$ states and a dark state $E_{i}^{+}$that can couple them.

\section{B. Wave-packet dynamics induced by XUV in the presence of a dressing field}

In this section we study the situation when the XUV pulse overlaps with a dressing NIR field that is a weak copy of the probe NIR field (i.e., the dressing field is to be carefully distinguished from the probe field). Being in temporal overlap with the XUV pulse, the NIR dressing field enables us to investigate the coherent coupling and wave-packet dynamics between both odd-parity and even-parity inner-shell-excited states $[59,60]$. The intensity of such a dressing NIR field usually reaches $1-5 \%$ of the delayed probe NIR field, which is sufficient to induce significant couplings, as demonstrated in the following.

Figure 4 shows the square root of the Fourier-transformed ATAS of $\mathrm{Xe}$ for a dressing field with an amplitude of $A_{d}=0.2 A_{I R}$. Note that $20 \%$ of the amplitude corresponds to $4 \%$ of the intensity. For the short pulses in Figs. 4(a) and 4(d) the diagonal features, corresponding to the dynamics inside the wave packet, are created by the XUV pulse in the presence of the dressing NIR field. Originally, the present dressing field
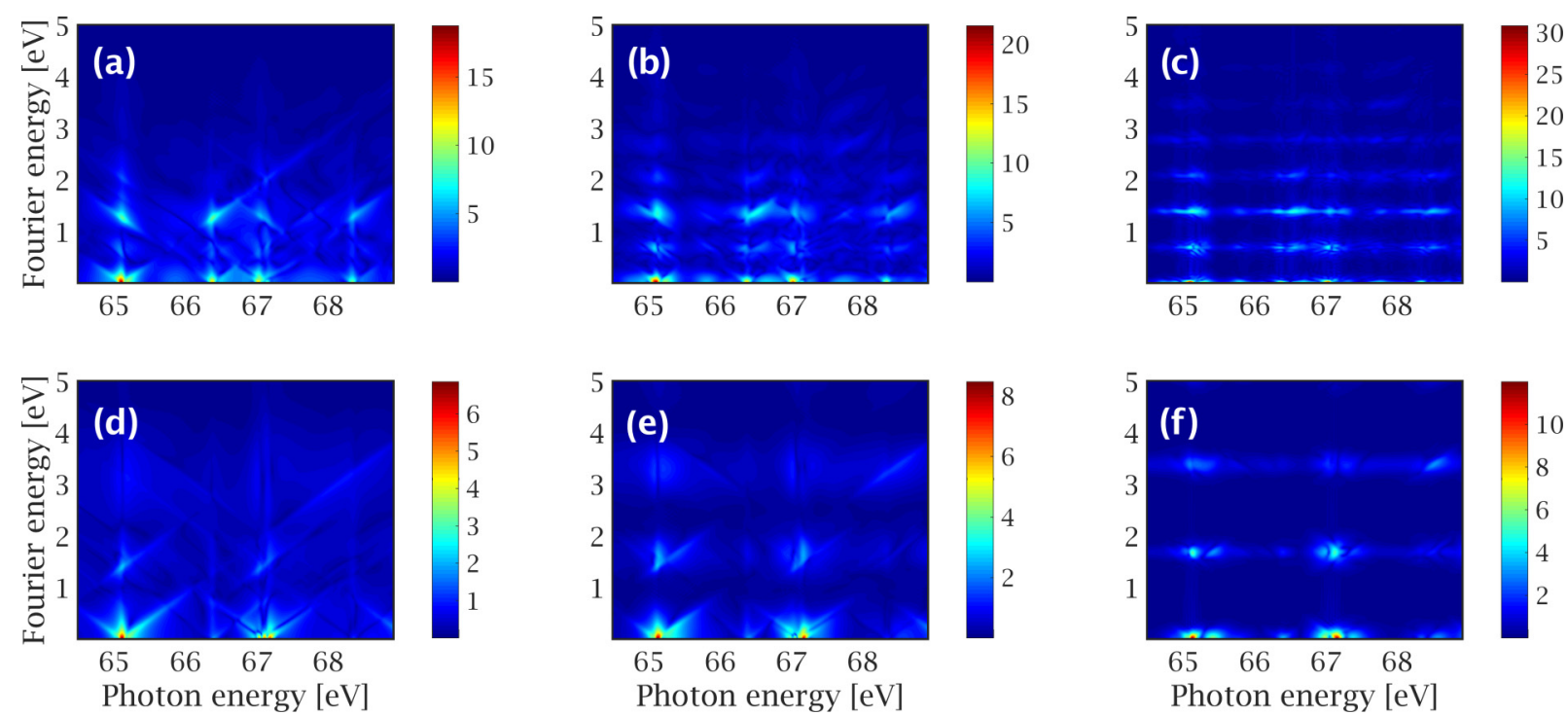

FIG. 4. Square root of the Fourier-transform amplitude of the XUV cross section of Xe $\left[\sqrt{\left|\tilde{\sigma}\left(\omega, \omega^{\prime}\right)\right|}\right]$ in the presence of a dressing field. The photon energy of the probe field is (a)-(c) $\omega_{I R}=0.7 \mathrm{eV}$ and (d)-(f) $\omega_{I R}=1.7 \mathrm{eV}$. The duration of the probe field is (a) and (d) one period of the field $T$, (b) and (e) two periods $2 T$, and (c) and (f) $6 T$. 

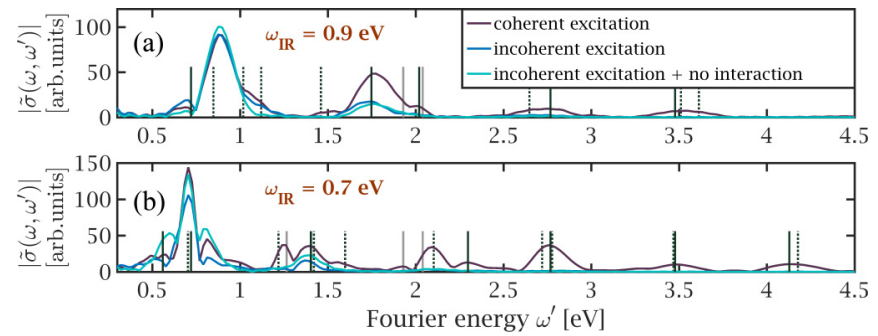

FIG. 5. Fourier-transformed XUV cross section in the presence of a dressing field with amplitude $A_{d}=0.2 A_{I R}$ for the $4 d_{5 / 2}^{-1} 6 p^{1}$ state with $\omega=65.1 \mathrm{eV}$ fixed. The duration of the laser pulses is $4 T$ and the photon energy $\omega_{I R}$ is (a) $0.9 \mathrm{eV}$ and (b) $0.7 \mathrm{eV}$. Gray solid lines mark bright-bright resonances, black solid lines mark bright-dark resonances, and black dashed lines indicate LIS-bright resonances.

provides a coupling with one photon $\omega_{I R}$ between the bright and dark states, such as bright state $4 d_{3 / 2}^{-1} 6 p^{1}\left(4 d_{5 / 2}^{-1} 6 p^{1}\right)$ and dark state $4 d_{3 / 2}^{-1} 6 s^{1}\left(4 d_{5 / 2}^{-1} 6 s^{1}\right)$. Although the intensity of the dressing field is weak, it leads to a very strong coupling in the case of $\omega_{I R}=1.7 \mathrm{eV}$. For example, the diagonal features around $1.3 \mathrm{eV}$ in Fig. 4(d) are more prominent than those in Fig. 2(d). For the long pulses in Figs. 4(c) and 4(f), the horizontal features emerge around 1.4 and $2.1 \mathrm{eV}$ in Fig. 4(c) and 1.7 and $3.4 \mathrm{eV}$ in Fig. 4(f), which correspond to $\omega^{\prime}=n \omega_{I R}$ $(n=1,2,3, \ldots)$.

To analyze these oscillations in more detail we present in Fig. 5 the Fourier-transformed ATAS at the main absorption peak $4 d_{5 / 2}^{-1} 6 p^{1}$ in the presence of the dressing field. The photon energy $\omega_{I R}$ and pulse duration are the same as used in Fig. 3. In contrast to Fig. 3, the resonances between bright states and dark states are visible (black solid lines), which are caused by the coherence between odd-parity and even-parity atomic eigenstates. The strongest oscillations have Fourier energies $\omega^{\prime}=n \omega_{I R}(n=1,2,3, \ldots)$ for both photon energies shown. The strength of these oscillations depends on whether the harmonics of $\omega_{I R}$ are energetically close enough to drive an interference between two excitation paths, as well as on the strength of the dipole moments of the states involved in the interaction.

\section{Experimental setup}

In the experiment, we use a helium-filled, differentially pumped hollow-core fiber (Femtolasers Kaleidoscope) and chirped mirrors (PC70, Ultrafast Innovations) to generate almost octave-spanning NIR pulses at a central photon energy of $\omega_{I R}=1.6 \mathrm{eV}$ and 1-mJ pulse energy from a commercial Ti:sapphire laser system (Femtolasers HE/HR). The pulses are characterized using the DScan technique [61,62], which reveals a pulse-intensity duration of $4.3 \mathrm{fs}$. The coherent XUV light is produced from high-harmonic generation (HHG) in neon driven by the NIR pulses and has a continuous spectrum over the range of 40-70 eV photon energy. The NIR and XUV pulses copropagate, and an aperture controls the NIR power without affecting the central XUV beam. Using a piezoelectrically driven split mirror, which is combined with a spatial filter unit, we introduce a time delay of sub-30-as stability between the NIR and XUV pulses. Afterward, both beams are reflected off a toroidal mirror, which refocuses the beams into the target gas cell, after which an XUV spectrometer is used to measure the spectrum of the transmitted XUV light with 45-meV resolution [63]. The thin-foil aluminum filters used in the spatial filter unit to block the NIR light are known to contain microholes that allow a small fraction of the NIR pulse to pass. This leakage is temporally locked to the XUV radiation and constitutes a weak replica of the main NIR pulse with typically a few percent of its power, realizing the dressing-pulse geometry as described in Sec. III B.

When the XUV pulse interacts with the xenon atom at photon energies above $65 \mathrm{eV}$, electronic transitions from the $4 d$ core shell to unoccupied $n p$ levels $(n \geqslant 6)$ are triggered (note that transitions to $n f$ levels are suppressed in $\mathrm{Xe}$ [64]). For each principal quantum number $n$, a fine-structure doublet of absorption lines is created due to spin-orbit interaction [65] of the hole. Here we focus on the absorption lines at 65.11 and $67.04 \mathrm{eV}$ for the case of $n=6$, because these are the strongest transitions of the series. These core-excited states decay rapidly via the Auger process, leading to lifetimes of less than $6.5 \mathrm{fs}$, which corresponds to natural linewidths of approximately $0.1 \mathrm{eV}$, respectively, with the natural line shape, to a good approximation, being described by a Lorentzian profile [54,65]. Compared to the 1.31-eV splitting of the $\mathrm{Xe}^{+} 5 p$ hole states that have also been encountered in previous experiments $[21,66,67]$, the spin-orbit splitting of these core-excited states is substantially stronger and amounts to $1.93 \mathrm{eV}$ (for $n \rightarrow \infty$, the energy splitting converges to $1.97 \mathrm{eV}$ [65]). Note that the above absorption lines correspond to the energies used in our calculation as listed in Table I $\left(4 d_{5 / 2}^{-1} 6 p^{1}\right.$ and $\left.4 d_{3 / 2}^{-1} 6 p^{1}\right)$.
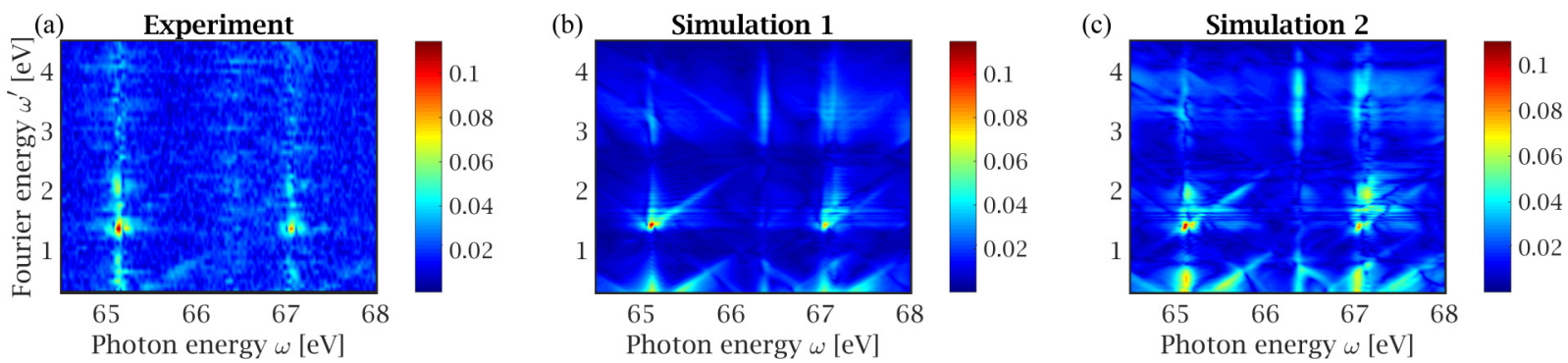

FIG. 6. Square root of (a) experimental and (b) and (c) simulated XUV spectra $\sqrt{\left|\tilde{\sigma}\left(\omega, \omega^{\prime}\right)\right|}$. In simulation 1, the nominal pulse shape from the experiment is used. In simulation 2 , a modified pulse shape is used as described in the text. 

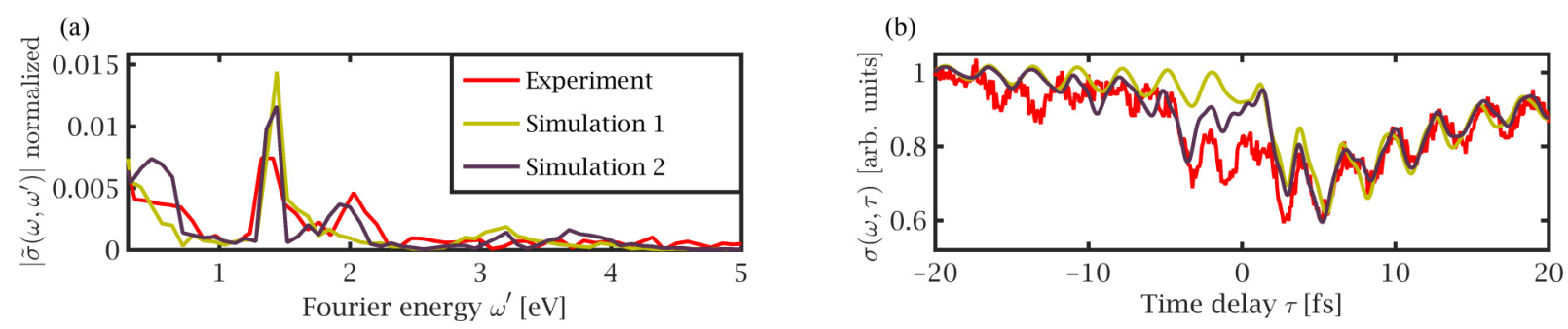

FIG. 7. Experimental and simulated XUV cross sections at $\omega=65.1 \mathrm{eV}$ in the (a) Fourier-energy and (b) time-delay domains.

\section{Comparison with experiment}

Figure 6 presents a comparison of the experimental and simulated Fourier-transformed XUV cross sections as a function of $\omega$ and $\omega^{\prime}$. A slice of the XUV cross section at $\omega=$ $65.1 \mathrm{eV}$ is plotted in the $\omega^{\prime}$ and $\tau$ domains in Fig. 7. In simulation 1 , we employ the laser pulse $\varepsilon(t)$ measured experimentally in front of the HHG gas cell. In simulation 2, we use a modified pulse with a much stronger 2-eV energy component (see a comparison of the pulse shapes in Fig. 8 and a description of how to construct the pulse shape at the end of this section), which gives partly better agreement with the experimental results. The intensity of the probe field defines the depth of the cross section $\sigma(\omega, \tau)$ attenuation [in Fig. 7(b)], and the intensities of the probe and dressing fields together determine the strength of oscillations with the time delay $\tau$. Guided by this correlation, we have chosen the strength of the probe field as $A_{I R}=0.0009$ and 0.0012 a.u. for simulations 1 and 2 , respectively. The intensity of the dressing pulse is $5 \%$ of the intensity of the probe laser field in both cases.

The diagonal streaks in Fig. 6(a) are reproduced in Figs. 6(b) and 6(c). All three panels in Fig. 6 show pronounced $4 d_{5 / 2}^{-1} 6 p^{1}(\omega=65.110 \mathrm{eV})$ and $4 d_{3 / 2}^{-1} 6 p^{1}(\omega=67.039 \mathrm{eV})$ peaks at $\omega^{\prime} \approx 1.4 \mathrm{eV}$. The associated time-domain dynamics are a consequence of dressing-field-mediated coupling between the $6 p$ and $6 s$ Rydberg-electron states. Without the dressing field, the 1.4-eV beating disappears. The oscillation at $\omega^{\prime} \approx 2 \mathrm{eV}$, which is observed in experiment, is not reproduced in simulation 1. Looking at the $\tau$ dependence of the XUV cross section in Fig. 7, we observe that the discrepancy between simulation 1 and experiment appears near $\tau=0$ and at negative delay, i.e., when the XUV pulse overlaps with or comes after the NIR pulse. When the XUV pulse comes first $(\tau>0)$, both simulations are in good agreement with experiment.

The discrepancies at negative $\tau$ might be an indication that the NIR field interacting with the Xe atoms differs from the

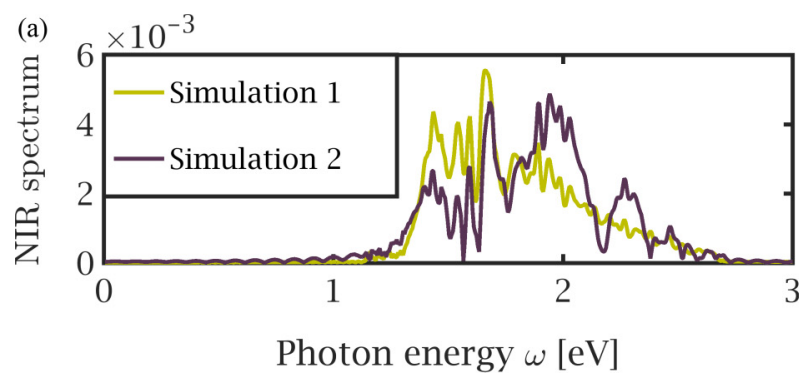

NIR field characterized in front of the HHG gas cell. This could be caused by laser field interaction with the optics or plasma in the HHG gas cell. Field ionizing media (FIM) are known to be able to add a chirp to the trailing part of a laser pulse [68]. Owing to the phase modulation induced by plasmas in FIM, the laser frequency increases with time (positive chirp) at the leading edge and then decreases (negative chirp) back to the original frequency in the remaining part of the pulse [69]. Guided by this, we apply the chirp effect to the experimentally measured spectral amplitude $A(\omega)$ and spectral phase $\phi(\omega)$,

$$
\varepsilon_{c h}^{ \pm}(\omega)=A(\omega) e^{-i \phi(\omega)} \exp \left\{ \pm i a \omega^{2}\right\} .
$$

Then we add together their inverse Fourier transforms $\varepsilon_{c h}^{ \pm}(t)$, applying the switching function $f(t)=\frac{1}{2}\left\{1+\frac{2}{\pi} \arctan \frac{t-b}{c}\right\}$,

$$
E_{c h}(t)=f(t) \varepsilon_{c h}^{-}(t)+\{1-f(t)\} \varepsilon_{c h}^{+}(t) .
$$

Here $a, b$, and $c$ are free parameters. The magnitude of the FIM-induced chirp depends on the properties of the HHG gas cell, including the percentage of ionization of the HHG target gas. If this phenomenon leads to an enhancement of the pulse spectrum in the vicinity of $2 \mathrm{eV}$, laser-mediated coupling to $4 d^{-1} n s^{1}(n>10)$ and $4 d^{-1} n d^{1}(n \geqslant 8)$ Rydberg states will be activated. In our simulation 2 we use the parameters $a=$ $15 \mathrm{fs}^{2}, b=0 \mathrm{fs}$, and $c=0.8 \mathrm{fs}$. This choice gives improved agreement with the experimental results and is consistent with Refs. [70,71], where a dynamic blueshift from a carrier wavelength of $750 \mathrm{~nm}(1.65 \mathrm{eV})$ to some $550 \mathrm{~nm}(2.25 \mathrm{eV})$ was observed in HHG in Ne. Even though our guess regarding the pulse-phase modifications in the HHG gas cell does not provide perfect agreement with experiment for the XUV cross section in the negative delay domain $(\tau<0)$, it reproduces the main features of the XUV cross section and gives fairly good agreement in the Fourier-energy domain (Figs. 7 and 8). This makes it plausible that the FIM-induced chirp effect may have affected the experimental data.

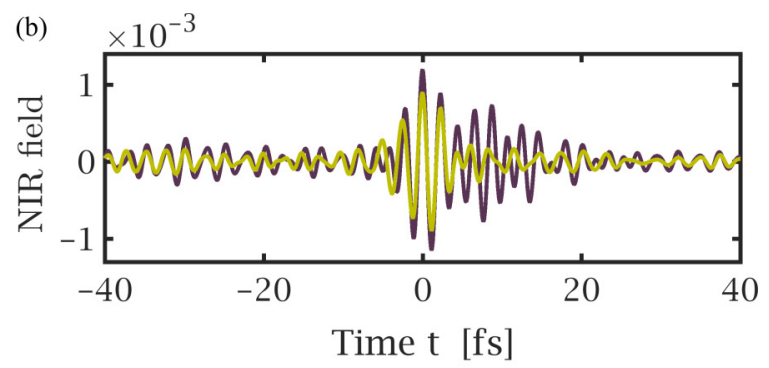

FIG. 8. Pulses used in simulations 1 and 2, in the (a) Fourier-energy and (b) time domains. 


\section{CONCLUSION}

We demonstrated that various aspects of coherent electron dynamics can be revealed when wave packets prepared by an attosecond pump pulse are probed using an NIR pulse. We found that inner-shell hole dynamics is much more difficult to detect than Rydberg-electron dynamics, because the dynamics of the inner-shell hole is defined mostly by the degree of coherence of the wave packet initially created by the XUV pulse, whereas Rydberg electrons get strongly dressed by the NIR pulse, thereby producing strong quantum coherence effects that are reflected in the ATAS. Typically, the transition dipole moment between states with different angular momentum $j$ of the hole is much weaker than for states with the same $j$. This makes the evolution of wave-packet components with different hole $j$ mostly independent of each other and thus not so sensitive to the presence of the probe field. Our analysis suggests that, in order to detect inner-shell hole dynamics using an NIR probe field, it is necessary to minimize the effect of the laser field on the Rydberg levels, which can be done by decreasing the field exposure time and by using fields that are far off-resonance.

We show that the presence of a dressing field or a slight modification of the spectrum of the probe pulse can dramatically change the simulated ATAS results. Thus, our work also suggests that a precise NIR pulse characterization in the target region behind the HHG gas cell is necessary for a qualitatively and quantitatively accurate interpretation of experimental ATAS data, due to the high sensitivity of Rydberg electrons to the NIR electric fields.

\section{ACKNOWLEDGMENT}

R.J. would like to thank the Helmholtz-OCPC Postdoc Program for support.
[1] J.-C. Diels and W. Rudolph, Ultrashort Laser Pulse Phenomena (Elsevier, Amsterdam, 2006).

[2] C. Rullière, T. Amand, and X. Marie, in Femtosecond Laser Pulses: Principles and Experiments, edited by C. Rullière (Springer, Cham, 1998), pp. 203-259.

[3] J. Manz and L. Wöste, Femtosecond Chemistry (VCH, Weinheim, 1995), Vol. 2.

[4] M. Lorenc, M. Ziolek, R. Naskrecki, J. Karolczak, J. Kubicki, and A. Maciejewski, Artifacts in femtosecond transient absorption spectroscopy, Appl. Phys. B 74, 19 (2002).

[5] N. Tamai and H. Miyasaka, Ultrafast dynamics of photochromic systems, Chem. Rev. 100, 1875 (2000).

[6] A. G. Joly and K. A. Nelson, Femtosecond transient absorption spectroscopy of chromium hexacarbonyl in methanol: Observation of initial excited states and carbon monoxide dissociation, J. Phys. Chem. 93, 2876 (1989).

[7] T. Pfeifer, C. Spielmann, and G. Gerber, Femtosecond x-ray science, Rep. Prog. Phys. 69, 443 (2006).

[8] A. H. Zewail, Femtochemistry: Atomic-scale dynamics of the chemical bond, J. Phys. Chem. A 104, 5660 (2000).

[9] L. Dhar, J. A. Rogers, and K. A. Nelson, Time-resolved vibrational spectroscopy in the impulsive limit, Chem. Rev. 94, 157 (1994).

[10] H. Petek and S. Ogawa, Femtosecond time-resolved two-photon photoemission studies of electron dynamics in metals, Prog. Surf. Sci. 56, 239 (1997).

[11] A. Othonos, Probing ultrafast carrier and phonon dynamics in semiconductors, J. Appl. Phys. 83, 1789 (1998).

[12] C. Voisin, N. Fatti, D. Christofilos, and F. Vallee, Ultrafast electron dynamics and optical nonlinearities in metal nanoparticles, J. Phys. Chem. B 105, 2264 (2001).

[13] A. Stolow, A. Bragg, and D. Neumark, Femtosecond timeresolved photoelectron spectroscopy, Chem. Rev. 104, 1719 (2004).

[14] K. Sokolowski-Tinten and D. Von Der Linde, Ultrafast phase transitions and lattice dynamics probed using laser-produced $\mathrm{x}$ ray pulses, J. Phys.: Condens. Matter 16, R1517 (2004).

[15] J. Güdde and U. Höfer, Femtosecond time-resolved studies of image-potential states at surfaces and interfaces of rare-gas adlayers, Prog. Surf. Sci. 80, 49 (2005).
[16] E. Nibbering, H. Fidder, and E. Pines, Ultrafast chemistry: Using time-resolved vibrational spectroscopy for interrogation of structural dynamics, Annu. Rev. Phys. Chem. 56, 337 (2005).

[17] P. Kukura, D. McCamant, and R. Mathies, Femtosecond stimulated Raman spectroscopy, Annu. Rev. Phys. Chem. 58, 461 (2007).

[18] M. Gühr, M. Bargheer, M. Fushitani, T. Kiljunen, and N. Schwentner, Ultrafast dynamics of halogens in rare gas solids, Phys. Chem. Chem. Phys. 9, 779 (2007).

[19] V. Sundstrom, Femtobiology, Annu. Rev. Phys. Chem. 59, 53 (2008).

[20] S. H. Southworth, D. A. Arms, E. M. Dufresne, R. W. Dunford, D. L. Ederer, C. Höhr, E. P. Kanter, B. Krässig, E. C. Landahl, E. R. Peterson, J. Rudati, R. Santra, D. A. Walko, and L. Young, $K$-edge x-ray-absorption spectroscopy of laser-generated $\mathrm{Kr}^{+}$ and $\mathrm{Kr}^{2+}$, Phys. Rev. A 76, 043421 (2007).

[21] Z.-H. Loh, M. Khalil, R. E. Correa, R. Santra, C. Buth, and S. R. Leone, Quantum State-Resolved Probing of StrongField-Ionized Xenon Atoms Using Femtosecond High-Order Harmonic Transient Absorption Spectroscopy, Phys. Rev. Lett. 98, 143601 (2007).

[22] L. Young, D. A. Arms, E. M. Dufresne, R. W. Dunford, D. L. Ederer, C. Höhr, E. P. Kanter, B. Krässig, E. C. Landahl, E. R. Peterson, J. Rudati, R. Santra, and S. H. Southworth, X-Ray Microprobe of Orbital Alignment in Strong-Field Ionized Atoms, Phys. Rev. Lett. 97, 083601 (2006).

[23] R. Santra, R. W. Dunford, and L. Young, Spin-orbit effect on strong-field ionization of krypton, Phys. Rev. A 74, 043403 (2006).

[24] P. Johnsson, J. Mauritsson, T. Remetter, A. L'Huillier, and K. J. Schafer, Attosecond Control of Ionization by Wave-Packet Interference, Phys. Rev. Lett. 99, 233001 (2007).

[25] T. Glover, M. Hertlein, S. Southworth, T. Allison, J. van Tilborg, E. Kanter, B. Krässig, H. Varma, B. Rude, R. Santra, A. Belkacem, and L. Young, Controlling x-rays with light, Nat. Phys. 6, 69 (2009).

[26] P. Ranitovic, X. Tong, B. Gramkow, S. De, B. DePaola, K. Singh, W. Cao, M. Magrakvelidze, D. Ray, I. Bocharova, H. Mashiko, A. Sandhu, E. Gagnon, M. Murnane, H. Kapteyn, I. Litvinyuk, and C. Cocke, IR-assisted ionization of helium 
by attosecond extreme ultraviolet radiation, New J. Phys. 12, 013008 (2010).

[27] M. Wickenhauser, J. Burgdörfer, F. Krausz, and M. Drescher, Time-Resolved Fano Resonances, Phys. Rev. Lett. 94, 023002 (2005).

[28] C. Buth, R. Santra, and L. Young, Electromagnetically Induced Transparency for X Rays, Phys. Rev. Lett. 98, 253001 (2007).

[29] T. Pfeifer, M. Abel, P. Nagel, A. Jullien, Z.-H. Loh, M. Bell, D. Neumark, and S. Leone, Time-resolved spectroscopy of attosecond quantum dynamics, Chem. Phys. Lett. 463, 11 (2008).

[30] R. Santra, C. Buth, E. Peterson, R. Dunford, E. Kanter, B. Krässig, S. Southworth, and L. Young, Strong-field control of x-ray absorption, J. Phys.: Conf. Ser. 88, 012052 (2007).

[31] C. Buth and R. Santra, Theory of x-ray absorption by laserdressed atoms, Phys. Rev. A 75, 033412 (2007).

[32] C. Buth and R. Santra, X-ray refractive index of laser-dressed atoms, Phys. Rev. A 78, 043409 (2008).

[33] A. McPherson, G. Gibson, H. Jara, U. Johann, T. Luk, I. McIntyre, K. Boyer, and C. Rhodes, Studies of multiphoton production of vacuum-ultraviolet radiation in the rare gases, J. Opt. Soc. Am. B 4, 595 (1987).

[34] M. Ferray, A. L'Huillier, X. F. Li, L. A. Lompre, G. Mainfray, and C. Manus, Multiple-harmonic conversion of $1064 \mathrm{~nm}$ radiation in rare gases, J. Phys. B 21, L31 (1988).

[35] P. B. Corkum, Plasma Perspective on Strong Field Multiphoton Ionization, Phys. Rev. Lett. 71, 1994 (1993).

[36] J. L. Krause, K. J. Schafer, and K. C. Kulander, High-Order Harmonic Generation from Atoms and Ions in the High Intensity Regime, Phys. Rev. Lett. 68, 3535 (1992).

[37] P. Paul, E. Toma, P. Breger, G. Mullot, F. Augé, P. Balcou, H. Muller, and P. Agostini, Observation of a train of attosecond pulses from high harmonic generation, Science 292, 1689 (2001).

[38] R. Santra, V. S. Yakovlev, T. Pfeifer, and Z.-H. Loh, Theory of attosecond transient absorption spectroscopy of strong-fieldgenerated ions, Phys. Rev. A 83, 033405 (2011).

[39] S. Pabst, A. Sytcheva, A. Moulet, A. Wirth, E. Goulielmakis, and R. Santra, Theory of attosecond transient-absorption spectroscopy of krypton for overlapping pump and probe pulses, Phys. Rev. A 86, 063411 (2012).

[40] E. Goulielmakis, Z.-H. Loh, A. Wirth, R. Santra, N. Rohringer, V. Yakovlev, S. Zherebtsov, T. Pfeifer, A. Azzeer, M. Kling, S. Leone, and F. Krausz, Real-time observation of valence electron motion, Nature (London) 466, 739 (2010).

[41] J. Mauritsson, T. Remetter, M. Swoboda, K. Klünder, A. L'Huillier, K. J. Schafer, O. Ghafur, F. Kelkensberg, W. Siu, P. Johnsson, M. J. J. Vrakking, I. Znakovskaya, T. Uphues, S. Zherebtsov, M. F. Kling, F. Lépine, E. Benedetti, F. Ferrari, G. Sansone, and M. Nisoli, Attosecond Electron Spectroscopy using a Novel Interferometric Pump-Probe Technique, Phys. Rev. Lett. 105, 053001 (2010).

[42] M. Holler, F. Schapper, L. Gallmann, and U. Keller, Attosecond Electron Wave-Packet Interference Observed by Transient Absorption, Phys. Rev. Lett. 106, 123601 (2011).

[43] C. Ott, A. Kaldun, L. Argenti, P. Raith, K. Meyer, M. Laux, Y. Zhang, A. Blättermann, S. Hagstotz, T. Ding, R. Heck, J. Madroñero, F. Martín, and T. Pfeifer, Reconstruction and control of a time-dependent two-electron wave packet, Nature (London) 516, 374 (2014).
[44] C. Ott, L. Aufleger, T. Ding, M. Rebholz, A. Magunia, M. Hartmann, V. Stooss, D. Wachs, P. Birk, G. D. Borisova, K. Meyer, P. Rupprecht, C. da Costa Castanheira, R. Moshammer, A. R. Attar, T. Gaumnitz, Z.-H. Loh, S. Düsterer, R. Treusch, J. Ullrich et al., Strong-Field Extreme-Ultraviolet Dressing of Atomic Double Excitation, Phys. Rev. Lett. 123, 163201 (2019).

[45] N. Shivaram, X.-M. Tong, H. Timmers, and A. Sandhu, Attosecond quantum-beat spectroscopy in Helium, J. Phys. B 49, 055601 (2016).

[46] A. Beck, B. Bernhardt, E. Warrick, M. Wu, S. Chen, M. Gaarde, K. Schafer, D. Neumark, and S. Leone, Attosecond transient absorption probing of electronic superpositions of bound states in neon: Detection of quantum beats, New J. Phys. 16, 113016 (2014).

[47] W. Cao, E. Warrick, D. Neumark, and S. Leone, Attosecond transient absorption of argon atoms in the vacuum ultraviolet region: Line energy shifts versus coherent population transfer, New J. Phys. 18, 013041 (2016).

[48] D. Kolbasova and R. Santra, Analytical theory of attosecond transient absorption spectroscopy of perturbatively dressed systems, Appl. Sci. 9, 1350 (2019).

[49] MATLAB, version 8.6.0.267246 (R2015b) (The Mathworks, Inc., Natick, 2015).

[50] E. Anderson, Z. Bai, C. Bischof, S. Blackford, J. Demmel, J. Dongarra, J. Du Croz, A. Greenbaum, S. Hammarling, A. McKenney, and D. Sorensen, LAPACK Users' Guide, 3rd ed. (Society for Industrial and Applied Mathematics, Philadelphia, 1999).

[51] M. F. Gu, The flexible atomic code, Can. J. Phys. 86, 675 (2008).

[52] G. C. King, M. Tronc, F. H. Read, and R. C. Bradford, An investigation of the structure near the $\mathrm{L}_{2,3}$ edges of argon, the $\mathrm{M}_{4,5}$ edges of krypton and the $\mathrm{N}_{4,5}$ edges of xenon, using electron impact with high resolution, J. Phys. B 10, 2479 (1977).

[53] M.-F. Lin, A. N. Pfeiffer, D. M. Neumark, S. R. Leone, and O. Gessner, Strong-field induced xuv transmission and multiplet splitting in $4 d^{-1} 6 p$ core-excited Xe studied by femtosecond xuv transient absorption spectroscopy, J. Chem. Phys. 137, 244305 (2012).

[54] S. Masui, E. Shigemasa, A. Yagishita, and I. A. Sellin, New measurements of the widths of the Xe 4d levels, J. Phys. B 28, 4529 (1995).

[55] S. Chen, M. J. Bell, A. R. Beck, H. Mashiko, M. Wu, A. N. Pfeiffer, M. B. Gaarde, D. M. Neumark, S. R. Leone, and K. J. Schafer, Light-induced states in attosecond transient absorption spectra of laser-dressed helium, Phys. Rev. A 86, 063408 (2012).

[56] S. Chen, M. Wu, M. B. Gaarde, and K. J. Schafer, Quantum interference in attosecond transient absorption of laser-dressed helium atoms, Phys. Rev. A 87, 033408 (2013).

[57] A. Beck, D. Neumark, and S. Leone, Probing ultrafast dynamics with attosecond transient absorption, Chem. Phys. Lett. 624, 119 (2015).

[58] M. Wu, S. Chen, S. Camp, K. Schafer, and M. Gaarde, Theory of strong-field attosecond transient absorption, J. Phys. B 49, 062003 (2016).

[59] T. Ding, C. Ott, A. Kaldun, A. Blättermann, K. Meyer, V. Stooss, M. Rebholz, P. Birk, M. Hartmann, A. Brown, H. V. D. 
Hart, and T. Pfeifer, Time-resolved four-wave-mixing spectroscopy for inner-valence transitions, Opt. Lett. 41, 709 (2016).

[60] N. Shivaram, H. Timmers, X.-M. Tong, and A. Sandhu, Attosecond-Resolved Evolution of a Laser-Dressed Helium Atom: Interfering Excitation Paths and Quantum Phases, Phys. Rev. Lett. 108, 193002 (2012).

[61] M. Miranda, T. Fordell, C. L. Arnold, A. L'Huillier, and H. Crespo, Simultaneous compression and characterization of ultrashort laser pulses using chirped mirrors and glass wedges, Opt. Express 20, 688 (2012).

[62] M. Miranda, C. L. Arnold, T. Fordell, F. Silva, B. Alonso, R. Weigand, A. L'Huillier, and H. Crespo, Characterization of broadband few-cycle laser pulses with the d-scan technique, Opt. Express 20, 18732 (2012).

[63] V. Stooß, M. Hartmann, P. Birk, G. D. Borisova, T. Ding, A. Blättermann, C. Ott, and T. Pfeifer, XUV-beamline for attosecond transient absorption measurements featuring a broadband common beam-path time-delay unit and in situ reference spectrometer for high stability and sensitivity, Rev. Sci. Instrum. 90, 053108 (2019).

[64] T. Koizumi, Y. Awaya, A. Fujino, Y. Itoh, M. Kitajima, T. Kojima, M. Oura, R. Okuma, M. Sano, T. Seikioka, N. Watanabe, and F. Koike, $4 d$ Photoionization of multiply charged $\mathrm{Xe}^{q+}(q=1-3)$ ions, Phys. Scr. 1997, 131 (1997).
[65] D. Ederer and M. Manalis, Photoabsorption of the $4 d$ electrons in xenon, J. Opt. Soc. Am. 65, 634 (1975).

[66] S. G. Sayres, E. R. Hosler, and S. R. Leone, Exposing the role of electron correlation in strong-field double ionization: X-ray transient absorption of orbital alignment in $\mathrm{Xe}^{+}$and $\mathrm{Xe}^{2+}$, J. Phys. Chem. A 118, 8614 (2014).

[67] M. Sabbar, H. Timmers, Y.-J. Chen, A. K. Pymer, Z.-H. Loh, S. G. Sayres, S. Pabst, R. Santra, and S. R. Leone, Stateresolved attosecond reversible and irreversible dynamics in strong optical fields, Nat. Phys. 13, 472 (2017).

[68] M. Geissler, G. Tempea, A. Scrinzi, M. Schnürer, F. Krausz, and T. Brabec, Light Propagation in Field-Ionizing Media: Extreme Nonlinear Optics, Phys. Rev. Lett. 83, 2930 (1999).

[69] J.-H. Kim and C. H. Nam, Plasma-induced frequency chirp of intense femtosecond lasers and its role in shaping highorder harmonic spectral lines, Phys. Rev. A 65, 033801 (2002).

[70] M. Hentschel, R. Kienberger, C. Spielmann, G. Reider, N. Milosevic, T. Brabec, P. Corkum, U. Heinzmann, M. Drescher, and F. Krausz, Attosecond metrology, Nature (London) 414, 509 (2001).

[71] M. B. Gaarde, M. Murakami, and R. Kienberger, Spatial separation of large dynamical blueshift and harmonic generation, Phys. Rev. A 74, 053401 (2006). 